\title{
Josephson point-contact resonance responses ${ }^{a)}$
}

\author{
Patrick S. Hagan ${ }^{\text {b) }}$ and Donald S. Cohen \\ Department of Applied Mathematics, California Institute of Technology, Pasadena, California 91125
}

(Received 31 August 1978; accepted for publication 4 December 1978)

\begin{abstract}
The voltage across a dc-current-driven Josephson point contact shunted by a resistive element with an applied small-amplitude ac signal current at frequency $\omega$ is calculated. First-order resonances are found to occur whenever $+\omega$ or $-\omega$ is near the Josephson frequency $\omega_{0}$. Second-order resonances occur whenever $\omega$ is near $\pm \omega_{0} / 2$. Singular perturbation techniques are used to obtain accurate approximate solutions for the contact voltage, in particular solutions when one of the above resonances occurs. These solutions all exhibit frequency (phase) locking when $\omega$ is near enough to $\pm \omega_{0}$ and $\pm \omega_{0} / 2$, and frequency pulling otherwise. The regions where frequency locking occurs are obtained.
\end{abstract}

PACS numbers: $74.30 . \mathrm{Gn}, \mathbf{7 4 . 5 0}+\mathrm{r}, \mathbf{8 5 . 2 5}+\mathrm{k}$

\section{INTRODUCTION}

Since the Josephson junction has many practical applications for the detection of weak high-frequency electromagnetic signals, quantitative analyses on sensitivity limits and relevant parameter groupings are very useful. Many investigators have used numerical ${ }^{1}$ and analog ${ }^{2}$ computational methods to provide quantitative explanations for experimental observations. Others have used analytic techniques, in particular regular perturbation methods, ${ }^{3}$ to obtain quantitative results. However, regular perturbation methods do not yield accurate quantitative results when resonance (and the corresponding effects of frequency locking and frequency pulling) occurs. In this paper we devise a singular perturbation technique (a multiscale perturbation expansion) and use it to obtain expressions for the contact voltage of a Josephson junction which are accurate even when resonance occurs.

Specifically, we use a multiscale perturbation technique to analyze the voltage response of a dc-current-driven Josephson point contact shunted by a resistive element to a weak applied ac signal current. In Sec. II we derive the circuit equation which governs the Josephson point contact. In Sec. III we analyze this circuit equation to first order. We find that the applied ac current resonates at first order when its frequency, $\omega$, is near $\pm \omega_{0}$, where $\omega_{0}$ is the Josephson frequency. Our perturbation technique is used to obtain an expression which accurately approximates the contact voltage for $\omega$ near $\omega_{0}$. In Sec. IV we examine this expression for the contact voltage. We find that the expression shows that frequency locking (alternatively called phase locking or entrainment) of the Josephson junction to the ac signal occurs whenever $\omega$ is in a small interval about $\omega_{0}$. Whenever $\omega$ is near to (but not in) this interval, the Josephson junction's oscillation frequency is "pulled" toward the signal frequency. Finally, when $\omega$ is not near $\omega_{0}$ the contact voltage expression reduces to the expression obtained by Kanter and Vernon $^{3}$ using regular perturbation methods. The interval in

\footnotetext{
"Supported in part by the U.S. Army Research Office (Durham) under Contract DAAG29-78-C-0011 and by the National Science Foundation under Grant MCS78-03036.

bsupported by the Fannie and John Hertz Foundation.
}

which frequency locking occurs is explicitly given. The resonance phenomena of frequency locking and frequency pulling have been experimentally observed. ${ }^{3}$

In Sec. $V$ we suppose that $\omega$ is not near $\pm \omega_{0}$ or zero. We analyze the circuit equation to second order. We find that second-order resonances occur whenever $\omega$ is near $\pm \frac{1}{2} \omega_{0}$. First we use our multiscale method to find the frequency shift when resonance does not occur. Then, we find an accurate expresssion for the contact voltages when $\omega$ is near $\frac{1}{2} \omega_{0}$. This expression exhibits frequency locking of the Josephson junction to the ac signal's second harmonic whenever $\omega$ is in an explicitly determined interval about $\frac{1}{2} \omega_{0}$, and frequency 'pulling' otherwise. In Ref. 3 this interaction of the harmonic of the signal with the junction has been described from experimental observations.

In Sec. VI we briefly discuss the frequencies $\omega$ for which higher-order resonances and consequent frequency locks occur.

\section{THE CIRCUIT EQUATION}

In this section we will derive the circuit equation which governs the Josephson point contact. The equivalent circuit of a current-driven Josephson point contact carrying both pair and quasiparticle current is shown in Fig. 1. Following the derivation of Kanter and Vernon ${ }^{3}$ we neglect all reactive elements in the circuit and all spatial variations of the pair current density. This yields a pair current

$$
I_{p}=I_{\mathrm{c}} \sin \left[\varphi\left(t^{\prime}\right)\right],
$$

with

$$
\varphi\left(t^{\prime}\right)=\frac{2 e}{\hbar} \int^{t^{\prime}} V(\tilde{t}) d \tilde{t},
$$

where $I_{c}$ is the critical super current, $V(\tilde{t})$ is the voltage across the contact, and $t^{\prime}$ is time. The circuit equation is

$$
V / R+I_{c} \sin \varphi=I_{B},
$$

in which $I_{B}$ is the battery current and $R$ is the normal leakage resistance. By introducing the dimensionless variables

$$
A=\frac{I_{B}}{I_{c}}, \quad v=\frac{V}{R I_{c}}, \quad t=\frac{2 e}{\hbar} R I_{c} t^{\prime},
$$




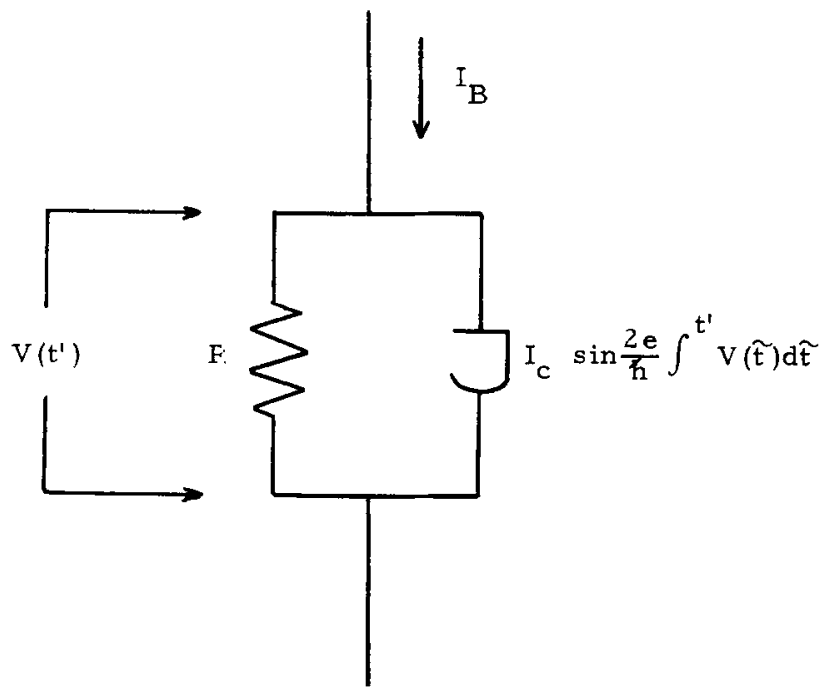

FIG. 1. Circuit of current-driven Josephson point contact.

one can write the circuit equation as

$$
\frac{d \phi}{d t}+\sin \phi=A \text {. }
$$

When a small ac signal current $I_{s} \sin (\omega t-\delta)$ is added, the circuit equation is clearly

$$
\frac{d \phi}{d t}+\sin \phi=A+\epsilon \sin (\omega t-\delta),
$$

in which $\epsilon \equiv I_{S} / I_{c}$ and $0<\epsilon \ll 1$ is assumed.

We will use singular perturbation techniques to study the behavior of solutions of this equation as $\epsilon$ and $\omega$ vary, in particular focusing our attention on the solutions when resonance occurs.

\section{FIRST-ORDER RESONANCES}

In this section we analyze the circuit equation, Eq. (6), to first order in $\epsilon$. We will find accurate expressions for $\phi$ in both the cases where resonance occurs and does not occur at this order.

In the limit that the small applied ac signal has zero amplitude the circuit equation reduces to

$$
\frac{d \phi}{d t}+\sin \phi=A
$$

It is easy to show that the general solution of Eq. (7) is

$$
\phi(t)=\Phi\left(t-t_{0}\right),
$$

where the function $\Phi(\theta)$ is defined by

$$
\tan \left[\frac{1}{2} \Phi(\theta)\right]=\left(\omega_{0} / A\right) \tan \left(\frac{1}{2} \omega_{0} \theta\right)+1 / A,
$$

where $\omega_{0} \equiv\left(A^{2}-1\right)^{1 / 2}$ is the Josephson frequency, and where $t_{0}$ is any constant. Note that we have assumed $A>1$; that is, the battery current is supracritical.

The applied ac signal is now assumed to have a small amplitude $\epsilon>0$. The usual method of solving for $\phi$ via a power series in $\epsilon$ cannot handle the cases where resonance occurs since then some terms in the power series are formally infinite. In order to be able to examine signals at resonant and near-resonant frequencies, we devise and utilize a multiscale perturbation method. In order to greatly simplify the algebra involved we immediately transform the circuit equation to obtain a new equation with simpler structure. Specifically, we define a new dependent variable $\theta(t)$ by the implicit relationship

$$
\phi(t) \equiv \Phi(\theta(t)),
$$

where $\Phi$ is the function defined in Eq. (9). We now switch from using $\phi$ as our dependent variable to using $\theta$. Substituting Eq. (10) into the circuit equation, Eq. (6), and using the fact that $\Phi$ has been defined so that

$$
\frac{d \Phi(\theta)}{d \theta}=A-\sin [\Phi(\theta)]
$$

one finds

$$
\frac{d \Phi}{d \theta} \frac{d \theta}{d t}=\frac{d \Phi}{d \theta}+\epsilon \sin (\omega t-\delta) .
$$

Differentiating Eq. (9) we obtain

$$
\frac{d \Phi}{d \theta}=\frac{\omega_{0}^{2}}{A+\cos \left(\omega_{0} \theta-\alpha\right)}
$$

with the constant $\alpha$ given by

$$
\sin \alpha=\omega_{0} / A, \quad \cos \alpha=1 / A .
$$

Thus Eq. (12) reduces to

$$
\begin{aligned}
\frac{d \theta}{d t}= & 1+\epsilon\left(\frac{A}{\omega_{0}^{2}} \sin (\omega t-\delta)+\frac{1}{2 \omega_{0}^{2}} \sin \left(\omega t+\omega_{0} \theta\right.\right. \\
& \left.-\alpha-\delta)+\frac{1}{2 \omega_{0}^{2}} \sin \left(\omega t-\omega_{0} \theta+\alpha-\delta\right)\right) .
\end{aligned}
$$

Although Eq. (14) looks complicated, the linearity of the equation at $\epsilon=0$ makes this equation much more practical than the original circuit equation for our purposes. Note that Eq. (14) is exact; no approximations have been introduced so far. This transformation was suggested by $\mathrm{Neu}^{4}$ who also used similar transformations of the dependent variable (so that the dominant equation has a simple structure) to solve a variety of problems.

We will now study Eq. (14) by devising a multiscale perturbation method. We define the slow time variable $T$ by

$$
T=\epsilon t
$$

and use the expansion

$\theta(\epsilon, t)=\theta^{(0)}(t, T)+\epsilon \theta^{(1)}(t, T)+\epsilon^{2} \theta^{(2)}(t, T)+\cdots$,

requiring that $\theta^{(1)}, \theta^{(2)}, \cdots$, are all of a size smaller than $O(1 / \epsilon)$ for all $t$. As usual we will use the extra freedom gained by allowing $\theta$ to depend on the long-time variable $T$ (as well as $t$ ) to enforce the requirement that the $\theta^{(i)}$ 's do not grow unreasonably large and thus invalidate the expansion of Eq. (16).

To proceed, we substitute $d / d t=\partial / \partial t+\epsilon \partial / \partial T$ and the expansion of Eq. (16) into our equation for $\theta$, Eq. (14). Equating the coefficients of like powers of $\epsilon$ yields the following hierarchy of equations:

$\frac{\partial \theta^{(0)}}{\partial t}=1$, 


$$
\begin{aligned}
\frac{\partial \theta^{(1)}}{\partial t} & \\
= & \frac{A}{\omega_{0}^{2}} \sin (\omega t-\delta)+\frac{1}{2 \omega_{0}^{2}} \sin \left(\omega t+\omega_{0} \theta^{(0)}-\alpha-\delta\right) \\
+ & \frac{1}{2 \omega_{0}^{2}} \sin \left(\omega t-\omega_{0} \theta^{(0)}+\alpha-\delta\right)-\frac{\partial \theta^{(0)}}{\partial T},(17 \mathrm{~b}) \\
\frac{\partial \theta^{(2)}}{\partial t}= & \frac{1}{2 \omega_{0}}\left[\cos \left(\omega t+\omega_{0} \theta^{(0)}-\alpha-\delta\right)\right. \\
& \left.-\cos \left(\omega t-\omega_{0} \theta^{(0)}+\alpha-\delta\right)\right] \theta^{(1)}-\frac{\partial \theta^{(1)}}{\partial T} .
\end{aligned}
$$

Equation (17a) clearly has the solution

$$
\theta^{(0)}=t+\beta(T),
$$

where the function $\beta(T)$ is undertermined at this stage of the perturbation procedure. Substituting expression (18a) into Eq. (17b) and integrating we find

$$
\begin{aligned}
\theta^{\prime \prime \prime}= & K^{(1)}-\frac{A}{\omega_{0}^{2} \omega} \cos (\omega t-\delta) \\
& -\frac{\cos \left[\left(\omega+\omega_{0}\right) t+\omega_{0} \beta-\alpha-\delta\right]}{2\left(\omega+\omega_{0}\right) \omega_{0}^{2}} \\
& -\frac{\cos \left[\left(\omega-\omega_{0}\right) t-\omega_{0} \beta+\alpha-\delta\right]}{2\left(\omega-\omega_{0}\right) \omega_{0}^{2}}-t \frac{d \beta}{d T},
\end{aligned}
$$

where $K^{\prime \prime}$ is a constant of integration and is set by the initial condition on $\theta$. One sees that if $\omega$ is not near $0,-\omega_{0}$, or $+\omega_{0}$ (i.e., if resonance does not occur), then the requirement that $\theta^{(1)}$ never gets unreasonably large implies $d \beta / d T=0$.

Hence, in this case $\beta(T)$ is some constant $\beta_{0}$, and thus an expression for $\phi$ accurate to order $\epsilon$ and for times $t$ of order $1 / \epsilon$ is given by

$$
\phi(\epsilon, \omega, t)=\Phi(\theta(\epsilon, \omega, t))
$$

with $\Phi(\theta)$ defined by

$$
\tan \left[\frac{1}{2} \Phi(\theta)\right]=\left(\omega_{0} / A\right) \tan \left(\frac{1}{2} \omega_{0} \theta\right)+1 / A
$$

and $\theta(\epsilon, \omega, t)$ given by

$$
\begin{aligned}
\theta(\epsilon, \omega, t)= & t+\beta_{0}+\epsilon\left(K^{(1)}-\frac{A}{\omega_{0}^{2} \omega} \cos (\omega t-\delta)\right. \\
& -\frac{\cos \left[\left(\omega+\omega_{0}\right) t+\omega_{0} \beta_{0}-\alpha-\delta\right]}{2\left(\omega+\omega_{0}\right) \omega_{0}^{2}} \\
& \left.-\frac{\left.\cos \left[\left(\omega-\omega_{0}\right) t-\omega_{0} \beta_{0}+\alpha-\delta\right)\right]}{2\left(\omega-\omega_{0}\right) \omega_{0}^{2}}\right) \\
& +O\left(\epsilon^{2}\right), \quad \omega \nsubseteq \pm \omega_{0}, \quad \omega £ 0 .
\end{aligned}
$$

This agrees with the solution of Kanter and Vernon ${ }^{3}$ to order $\epsilon$. We will briefly discuss this solution for the nonresonance case in Sec. IV.

We now examine the resonant situation. Specifically, suppose that $\omega \approx \omega_{0}$. We define $\eta$ by

$$
\eta \equiv\left(\omega-\omega_{0}\right) / \epsilon \text {. }
$$

Substituting this relationship [and Eq. (18a)] into Eq. (17b), we find

$$
\begin{aligned}
\frac{\partial \theta^{(1)}}{\partial t}= & \frac{A}{\omega_{0}^{2}} \sin \left(\omega_{0} t+\eta T-\delta\right)+\frac{1}{2 \omega_{0}^{2}} \sin \left[2 \omega_{0} t+\eta T\right. \\
& \left.+\omega_{\mathrm{d}} \beta(T)-\alpha-\delta\right]+\frac{1}{2 \omega_{0}^{2}} \sin \left(\eta T-\omega_{0} \beta+\alpha\right.
\end{aligned}
$$

$$
-\delta)-\frac{d \beta}{d T} .
$$

Integration yields

$$
\begin{aligned}
\theta^{(1)}= & K^{\prime \prime}-\frac{A}{\omega_{0}^{3}} \cos \left(\omega_{0} t+\eta T-\delta\right) \\
& -\frac{\cos \left[2 \omega_{0} t+\eta T+\omega_{\alpha} \beta(T)-\alpha-\delta\right]}{4 \omega_{0}^{3}} \\
& +t\left(\frac{1}{2 \omega_{0}^{2}} \sin \left(\eta T-\omega_{0} \beta+\alpha-\delta\right)-\frac{d \beta}{d T}\right),
\end{aligned}
$$

where again $K^{\prime \prime \prime}$ is an integration constant. Since $\theta^{\prime \prime}$ is required to remain reasonably sized for all $t$, we conclude that

$$
\frac{d \beta}{d T}=\frac{1}{2 \omega_{0}^{2}} \sin \left(\eta T-\omega_{0} \beta+\alpha-\delta\right) .
$$

Thus, in this resonant case we find that to leading order in $\epsilon$ the solution is $\theta=t+\beta(T)$, where $\beta$ evolves according to Eq. (21). Equation(21) contains all the pertinent information about the $O(1)$ effects caused by the small resonant signal. We now solve Eq. (21).

Define $P$ by

$$
P(T)=\eta T-\omega_{\delta} \beta(T)+\alpha-\delta .
$$

In terms of $P$ our secular equation, Eq. (21), is

$$
\frac{d P}{d T}=\eta-\frac{1}{2 \omega_{0}} \sin P,
$$

which has for its solutions

$$
\begin{aligned}
\tan \left[\frac{1}{2} P(T)\right]= & \frac{\left(\gamma^{2}-1\right)^{1 / 2}}{\gamma} \tan \left(\frac{\left(\gamma^{2}-1\right)^{1 / 2}}{4 \omega_{0}}\left(T-T_{0}\right)\right) \\
& +\frac{1}{\gamma} \quad \text { if } \gamma^{2} \geqslant 1 \\
\tan \left[\frac{1}{2} P(T)\right]= & -\frac{\left(1-\gamma^{2}\right)^{1 / 2}}{\gamma} \tanh \left(\frac{\left(1-\gamma^{2}\right)^{1 / 2}}{4 \omega_{0}}\left(T-T_{0}\right)\right) \\
& +\frac{1}{\gamma} \quad \text { if } \gamma^{2} \leqslant 1
\end{aligned}
$$




$$
\begin{aligned}
\tan \left[\frac{1}{2} P(T)\right]= & -\frac{\left(1-\gamma^{2}\right)^{1 / 2}}{\gamma} \operatorname{ctnh}\left(\frac{\left(1-\gamma^{2}\right)^{1 / 2}}{4 \omega_{0}}\left(T-T_{0}\right)\right) \\
& +\frac{1}{\gamma} \quad \text { if } \gamma^{2} \leqslant 1
\end{aligned}
$$

in which the definition

$$
\gamma \equiv 2 \eta \omega_{0} \equiv \frac{2\left(\omega-\omega_{0}\right)}{\epsilon} \omega_{0}
$$

has been used. Note that the initial value of $P$ (of $\beta$ ) determines not only the integration constant $T_{0}$ but also whether Eq. (24b) or ( $24 c)$ is to be used when $\gamma^{2} \leqslant 1$. Thus, in this case of $\omega \approx \omega_{0}$, our expression for the resonance solution is

$$
\begin{aligned}
& \phi(\epsilon, \omega, t)=\Phi(\theta(\epsilon, \omega, t)), \\
& \tan \left[\frac{1}{2} \Phi(\theta)\right]=\frac{\omega_{0}}{A} \tan \left(\frac{\omega_{0}}{2} \theta\right)+\frac{1}{A},
\end{aligned}
$$

where now $\theta(\epsilon, \omega, t)$ is given by

$\theta(\epsilon, \omega, t)$

$$
\begin{aligned}
& =t+\beta(T)+\epsilon\left(K^{\prime 1)}-\frac{A}{\omega_{0}^{3}} \cos \left(\omega_{0} t+\eta T-\delta\right)\right. \\
& \left.-\frac{\cos \left[2 \omega_{0} t+\eta T+\omega_{0} \beta(T)-\alpha-\delta\right]}{4 \omega_{0}^{3}}\right)+O\left(\epsilon^{2}\right),
\end{aligned}
$$

and

$$
\beta(T)=-\frac{1}{\omega_{0}}[P(T)-\alpha+\delta-\eta T],
$$

with $P(T)$ given by Eqs. (24). This resonance solution is accurate to order $\epsilon$ and for times of order $1 / \epsilon$. In Sec. IV we discuss this solution. In particular, we will find that as $\gamma^{2}$ becomes large (as $\omega$ shifts away from $\omega_{0}$ ) this expression reverts to the expression found for the nonresonant case given by Eqs. (19). As $\gamma^{2}$ approaches unity we will find that frequency "pulling" occurs, and when $\gamma^{2} \leqslant 1$ frequency locking takes place.

The other two cases where first-order resonance occurs $\left(\omega \approx-\omega_{0}\right.$ and $\omega \approx 0$ ) can be treated in the same manner as the $\omega \approx \omega_{0}$ case. In particular, if $\omega \approx-\omega_{0}$ we define

$$
\tilde{\eta}=-\frac{\omega+\omega_{0}}{\epsilon}, \quad \tilde{\gamma}=2 \omega_{0} \tilde{\eta}
$$

and the resulting expression for the resonance solution is $\theta(\epsilon, \omega, t)$

$$
\begin{aligned}
& =t+\beta(T)+\epsilon\left(K^{(1)}+\frac{A}{\omega_{0}^{3}} \cos \left(\omega_{0} t+\tilde{\eta} T+\delta\right)\right. \\
& \left.+\frac{\cos \left[2 \omega_{0} t+\tilde{\eta} T+\omega_{0} \beta(T)-\alpha+\delta\right]}{4 \omega_{0}^{3}}\right)+O\left(\epsilon^{2}\right),
\end{aligned}
$$

$\beta(T)=\frac{1}{\omega_{0}}[\widetilde{P}(-T)+\alpha+\delta+\tilde{\eta} T]$, where $\widetilde{P}(T)$ is given by Eqs. (24) with $P$ and $\gamma$ replaced by $\widetilde{P}$ and $\tilde{\gamma}$. For the $\omega \approx 0$ case we define

$$
\tilde{\tilde{\eta}}=\omega / \epsilon
$$

and the resulting resonance solution is

$$
\begin{aligned}
\theta(\epsilon, \omega, t)=t & +\beta(T)+\epsilon\left(K^{\prime \prime}+\frac{\sin \left[\omega_{0} t-\alpha+\omega_{0} \beta(T)\right]}{\omega_{0}^{3}}\right. \\
& \times \sin (\tilde{\eta} T-\delta))+O\left(\epsilon^{2}\right),
\end{aligned}
$$

$\beta(T)=\frac{A}{\omega_{0}^{2} \tilde{\eta}}\left\{1-\cos \left[\tilde{\tilde{\eta}}\left(T-T_{0}\right)-\delta\right]\right\}$.

Of those two cases, $\omega=-\omega_{0}$ represents a resonant situation entirely analogous to the $\omega=\omega_{0}$ case, and the discussion in Sec. IV about the nature of the $\omega=\omega_{0}$ solution carries over to the $\omega=-\omega_{0}$ case as well. The case of $\omega \approx 0$, however, can be viewed as the battery current being $A+\epsilon \sin (\tilde{\eta} T-\delta)$, and thus fluctuating on the adiabatically long time scale of $\tilde{\eta} T$. In this light, Eqs. (30) are seen to represent the firstorder effects of the slow variations in the Josephson frequency $\omega_{0}(T) \equiv\left\{[A+\epsilon \sin (\omega t-\delta)]^{2}-1\right\}^{1 / 2}$.

\section{NATURE OF THE RESONANCE SOLUTIONS}

In this section we will discuss the qualitative nature of the resonance solutions found in the previous section. Since the cases $\omega \approx+\omega_{0}$ and $\omega \approx-\omega_{0}$ are very similar, we will deal directly only with the case $\omega \approx+\omega_{0}$, and note that virtually identical considerations hold for the $\omega \approx-\omega_{0}$ case. In order to clearly distinguish the phenomena caused by resonance, we will also briefly discuss the non-resonance solution found in section III. A fuller accounting of the nonresonance case can be found in Ref. [3].

The dependent variable $\theta$ is very convenient to work with mathematically. However, it is not readily physically interpretable. Thus we will discuss the resonance (and nonresonance) solutions in terms of the more physically significant variable $v \equiv d \phi / d t$, which is the contact voltage in reduced units. Since we have

$$
\begin{aligned}
& v=\frac{d \phi}{d t}=\Phi^{\prime}(\theta) \frac{d \theta}{d t} \\
& =\left[\Phi^{\prime}\left(\theta^{(0)}\right)+\epsilon \Phi^{\prime \prime}\left(\theta^{(0)}\right) \theta^{(1)}+\cdots\right] \\
& \times\left[\frac{\partial \theta^{(0)}}{\partial t}+\epsilon\left(\frac{\partial \theta^{(0)}}{\partial T}+\frac{\partial \theta^{(1)}}{\partial t}\right)+\cdots\right], \\
& v=v^{(0)}+\epsilon v^{(1)}+\epsilon^{2} v^{(2)}+\cdots, \\
& v^{(0)}=\frac{\omega_{0}^{2}}{A+\cos \left[\omega_{0} t+\omega_{\alpha} \beta(T)-\alpha\right]}, \\
& v^{(1)}=\frac{\omega_{0}^{2}}{A+\cos \left[\omega_{0} t+\omega_{0} \beta(T)-\alpha\right]}\left(\frac{d \beta}{d T}+\frac{\partial \theta^{(1)}}{\partial t}\right) \\
& +\frac{\omega_{0}^{3} \sin \left[\omega_{0} t+\omega_{0} \beta(T)-\alpha\right]}{\left[A+\cos \left(\omega_{0} t+\omega_{\alpha} \beta(T)-\alpha\right]^{2}\right.} \theta^{\prime 1)},
\end{aligned}
$$


where $\theta^{(0)}+\epsilon \theta^{(1)}$ is given by Eq. (19c) for the nonresonance case and by Eqs. (26c) and (26d) in the resonance case $\omega \approx \omega_{0}$. We will focus our attention on the two key quantities of the first-order voltage response $\epsilon v^{(1)}$ and the phase $\chi$ of the dominant term in the voltage expansion, which is given by

$$
\chi=\omega_{0} t+\omega_{0} \beta(T)-\alpha .
$$

We now consider the nonresonance case. In this case $\theta^{(0)}$ and $\theta^{(1)}$ are given in Eq. (19c) and $\beta(T)$ is the constant $\beta_{0}$. The first-order voltage response is

$$
\epsilon v^{\prime \prime \prime}(t)=\frac{\partial}{\partial t} F
$$

where $F(t)$ is defined by

$$
\begin{aligned}
F= & \left(\omega_{0}^{2} K^{\prime \prime \prime}-\frac{A}{\omega} \cos (\omega t-\delta)\right. \\
& -\frac{\cos \left[\left(\omega+\omega_{0}\right) t+\omega_{0} \beta_{0}-\alpha-\delta\right]}{2\left(\omega+\omega_{0}\right)} \\
& \left.-\frac{\cos \left[\left(\omega-\omega_{0}\right) t-\omega_{0} \beta_{0}+\alpha-\delta\right]}{2\left(\omega-\omega_{0}\right)}\right) \\
& \times\left[A+\cos \left(\omega_{0} t+\omega_{\alpha} \beta_{0}-\alpha\right)\right]^{-1} .
\end{aligned}
$$

Thus, this voltage response varies over time in a complicated manner. However, averaging this first-order response voltage over $t$ yields

$$
\begin{aligned}
\overline{\epsilon v^{(1)}} & \equiv \lim _{t \rightarrow \infty}(1 / t) \int_{0}^{t} \epsilon v^{(1)}(\tilde{t}) d \tilde{t} \\
& =\lim _{t \rightarrow \infty}(1 / t)[F(t)-F(0)]=0 .
\end{aligned}
$$

This last step follows from the boundedness of $F$. Thus the time averaged first-order voltage response is zero. The phase of the dominant voltage term is, from Eq. (34),

$$
\chi=\omega_{0} t+\omega_{0} \beta_{0}-\alpha,
$$

and clearly the dominant voltage oscillates at precisely the Josephson frequency $\omega_{0}$.

We now consider the resonant case $\omega \approx \omega_{0}$. If we substitute the expressions in Eqs. (26c) and (26d) for $\theta^{(0)}, \theta^{(1)}$, and $\beta(T)$ into Eq. (33b) we find that the first-order response voltage, $\epsilon v^{(1)}(t, T)$, varies in a complicated manner over $t$ and $T$. However, if we average $\epsilon v^{(1)}$ over the fast time variable $t$ and denote the result by $\overline{\epsilon v^{(1)}}(T)$, we find

$$
\begin{aligned}
\overline{\epsilon v^{(1)}}(T) & \equiv \lim _{t \rightarrow \infty}(1 / t) \int_{0}^{t} v^{(1)}(\tilde{t}, T) d \tilde{t} \\
& =\frac{\epsilon}{2 \omega_{0}} \sin \left[\eta T-\omega_{0} \beta(T)+\alpha-\delta\right],
\end{aligned}
$$

where $\eta \equiv\left(\omega-\omega_{0}\right) / \epsilon$ as before, and $\beta(T)$ is given in Eqs. (24) and (26d). We now average over $T$ also, defining $\overline{\epsilon v^{(1)}}=\lim (1 / T) \int_{0}^{T \epsilon v^{(1)}}(\widetilde{T}) d \widetilde{T}$ as $T \rightarrow \infty$. The result is

$$
\begin{array}{ll}
\overline{\epsilon v^{(1)}}=\epsilon \operatorname{sgn}(\gamma) \frac{|\gamma|-\left(\gamma^{2}-1\right)^{1 / 2}}{2 \omega_{0}}, & \gamma^{2} \geqslant 1 \\
\overline{\epsilon v^{(1)}}=\frac{\epsilon \gamma}{2 \omega_{0}}, & \gamma^{2} \leqslant 1,
\end{array}
$$

in which $\gamma \equiv 2 \omega_{0} \eta=2 \omega_{0}\left(\omega-\omega_{0}\right) / \epsilon$. Note that as $|\gamma|$ gets large (i.e., as we depart from resonance), the averaged firstorder response voltage is given asymptotically by $\overline{\epsilon v^{(1)}}$ $\sim \epsilon / 4 \omega_{0} \gamma$. Thus as $\gamma$ becomes large, the averaged voltage goes to zero-which is the nonresonance averaged voltage. more interesting resonance behavior. Substituting for $\beta$ in Eq. (34), we find that

$$
\chi=\omega_{0} t+\eta T-P(T)-\delta,
$$

and since $\eta T=\epsilon \eta t=\left(\omega-\omega_{0}\right) t$, we find

$$
\chi=\omega t-P(T)-\delta \text {. }
$$

Thus we conclude that when $\gamma^{2} \leqslant 1$; that is, when $\left|\omega-\omega_{0}\right|<\epsilon / 2 \omega_{0}$, the Josephson junction is frequency locked to (alternatively phase locked to or entrained by) the ac signal. This is because when $\gamma^{2} \leqslant 1$, the fact that tanh and ctnh both have the limiting value 1 as $T \rightarrow \infty$ implies that $P(T)$ approaches a limiting value as $T \rightarrow \infty$, and thus $\chi$ goes to $\chi=\omega t+$ const as $T$ gets large. In fact, if we define the instantaneous frequency as $d \chi / d t=\partial \chi / \partial t+\epsilon \partial \chi / \partial T$, then

$$
\begin{aligned}
\frac{d \chi}{d t}= & \omega+\epsilon \frac{1-\gamma^{2}}{2 \omega_{0}} \\
& \times\left\{\gamma \pm \cosh \left[\frac{\left(1-\gamma^{2}\right)^{1 / 2}}{2 \omega_{0}}\left(T-T_{0}\right)-\alpha^{\prime}\right]\right\}^{-1},
\end{aligned}
$$

where the constant $\alpha^{\prime}$ has been defined by

$$
\cosh \alpha^{\prime}=\frac{1}{\gamma}, \quad \sinh \alpha^{\prime}=\frac{\left(1-\gamma^{2}\right)^{1 / 2}}{\gamma},
$$

and the plus sign (minus sign) is taken when $P$ is given by Eq. (24b) [Eq. (24c)]. Thus the frequency locking of the junction occurs exponentially in $T=\epsilon t$.

When $\gamma^{2}>1$, the instantaneous frequency is

$$
\begin{aligned}
\frac{d \chi}{d t}= & \omega_{0}+\epsilon\left(\frac{\gamma}{2 \omega_{0}}-\frac{\gamma^{2}-1}{2 \omega_{0}}\right. \\
& \left.\times\left\{\gamma+\cos \left[\frac{\left(\gamma^{2}-1\right)^{1 / 2}}{2 \omega_{0}}\left(T-T_{0}\right)-\alpha^{\prime \prime}\right]\right\}^{-1}\right),
\end{aligned}
$$

where the constant $\alpha^{\prime \prime}$ has been defined by

$$
\cos \alpha^{\prime \prime}=\frac{1}{\gamma}, \quad \sin \alpha^{\prime \prime}=\frac{\left(\gamma^{2}-1\right)^{1 / 2}}{\gamma} .
$$

Thus, in this case, the instantaneous frequency oscillates on the $T=\epsilon t$ time scale with period $4 \pi \omega_{0} /\left(\gamma^{2}-1\right)^{1 / 2}$ (in units of $T$ ). Since the time-averaged instantaneous frequency is 


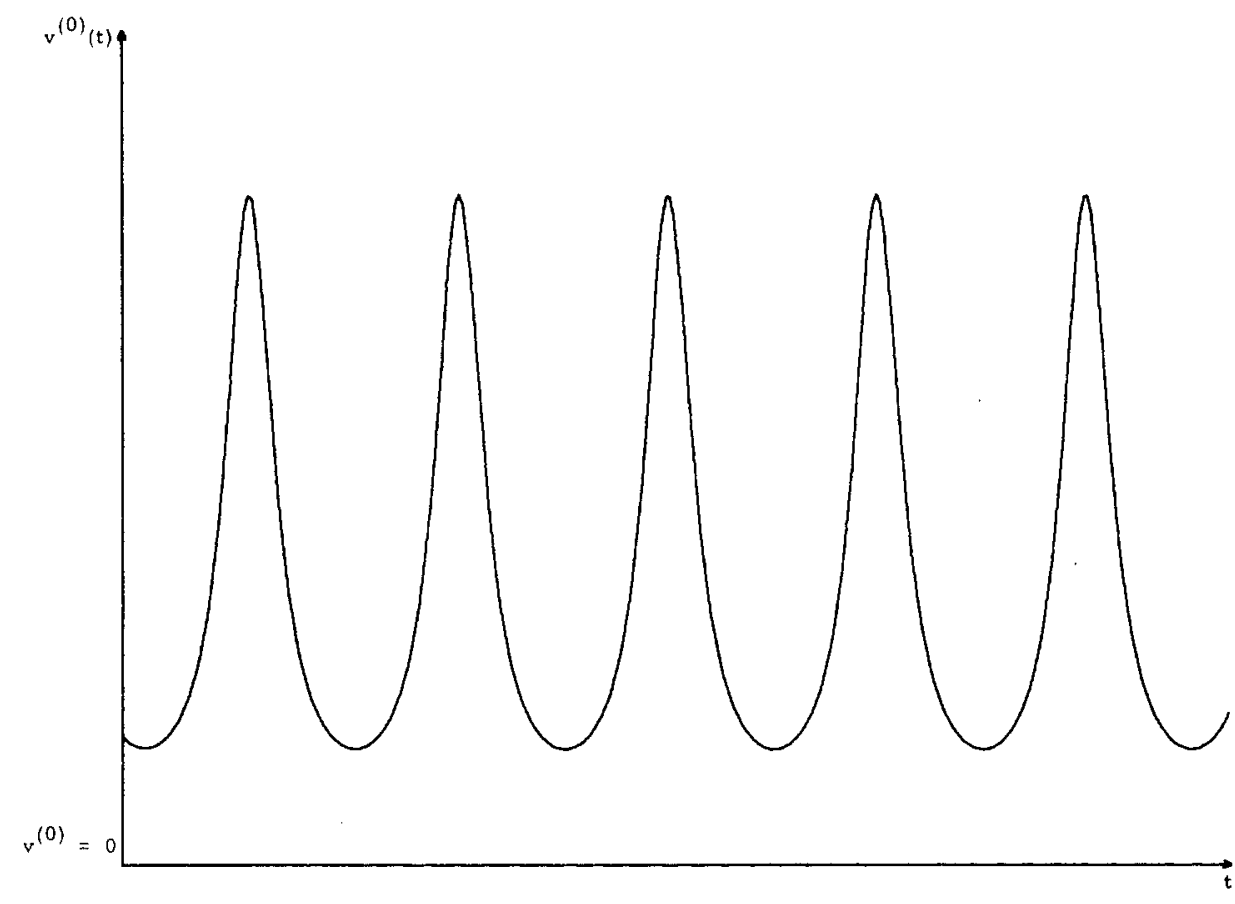

FIG. 2. A typical primary voltage oscillation $v^{\prime \prime \prime}(t)$ of the junction when resonance does not occur.

$$
\frac{\overline{d \chi}}{d t}=\omega_{0}+\frac{\epsilon}{2 \omega_{0}}(\operatorname{sgn} \gamma)\left[|\gamma|-\left(\gamma^{2}-1\right)^{1 / 2}\right]
$$

which has the limits $\omega_{0}+\left(\epsilon / 2 \omega_{0}\right) \gamma=\omega$ as $\gamma \rightarrow \pm 1$ and the limit $\omega_{0}$ as $\gamma^{2} \rightarrow \infty$, the interpretation of frequency pulling of the junction by the ac signal seems natural when $\gamma^{2}>1$. Note also that since the nonresonant solution has instantaneous frequency $\omega_{0}$, the average instantaneous frequency goes smoothly over into the nonresonance value as $\gamma^{2} \rightarrow \infty$.

To summarize this section, we found that in the nonresonance case the averaged first-order voltage response, $\overline{\epsilon v^{(1)}}$, is zero and the instantaneous frequency of the dominant voltage oscillation, $d \chi / d t$, is exactly the Josephson frequency $\omega_{0}$. In Figs. 2 and 3 we have graphed the dominant voltage oscillation $v^{(0)}(t)$ and the first-order voltage response $e v^{(1)}(t)$ for the nonresonance case. As $\omega$ approaches $\omega_{0}$, resonance phenomena begin to appear. Namely, as $\omega$ approaches $\omega_{0}$, $\epsilon v^{(1)}$ grows according to the formula $\omega-\omega_{0}$ $-\operatorname{sgn}\left(\omega-\omega_{0}\right)\left[\left(\omega-\omega_{0}\right)^{2}-\epsilon^{2} / 4 \omega_{0}^{2}\right]^{1 / 2}$ and $d \chi / d t$ acquires a small slow oscillation about its mean value of $\omega_{0}+\left\{\left(\omega-\omega_{0}\right)-\operatorname{sgn}\left(\omega-\omega_{0}\right)\left[\left(\omega-\omega_{0}\right)^{2}-\epsilon^{2} / 44 \omega_{0}^{2}\right]^{1 / 2}\right\}$.

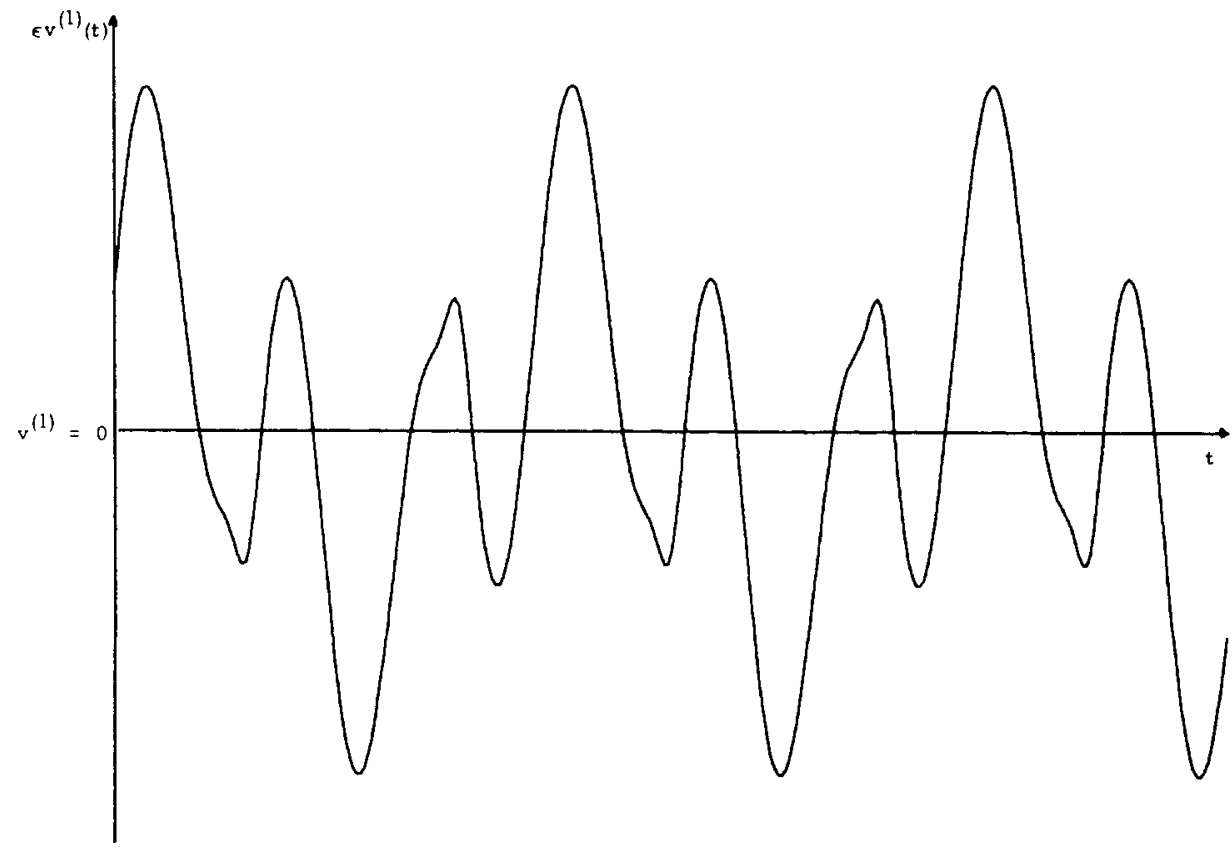

FIG. 3. A typical first-order response voltage of the junction when resonance does not occur. Verticle scale greatly magnified. 


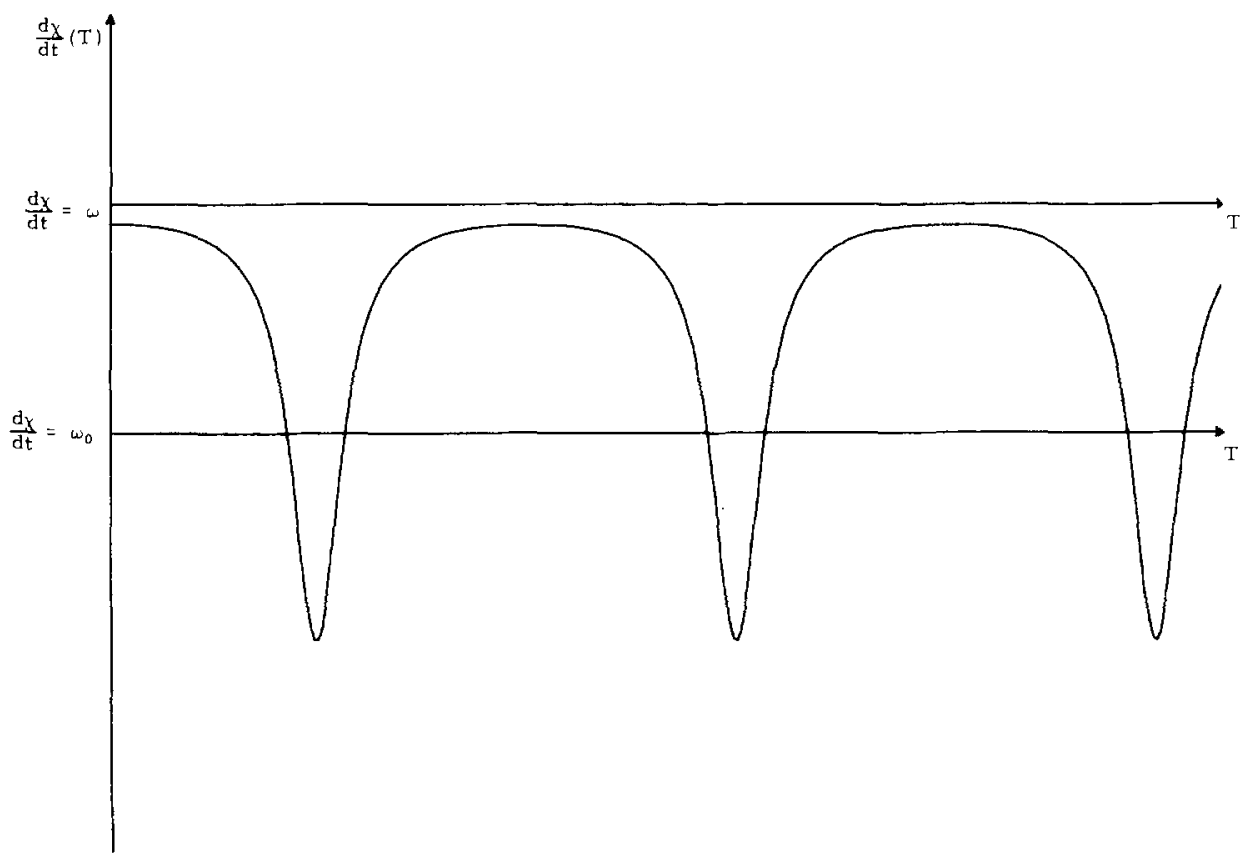

FIG. 4. The instantaneous frequency $d \chi / d t$ of the junction as a function of the slow time variable $T=\epsilon t$. Here the ac signal frequency $\omega$ is very near to (but outside of) the locking region $\left|\omega-\omega_{i}\right|<\epsilon / 2 \omega_{1}$

As $\omega$ nears $\omega_{0}$, the period of the oscillation of $d \chi / d t$ increases becoming infinite when $\left|\omega-\omega_{0}\right|=\epsilon / 2 \omega_{0}$. When $\omega$ is near enough to $\omega_{0}$ (when $\left.\left|\omega-\omega_{0}\right|<\epsilon / 2 \omega_{0}\right), \overline{\epsilon v^{(1)}}$ is given by $\overline{\epsilon v^{(1)}}$ $=\omega-\omega_{0}$ and the quantity $d \chi / d t$ locks exponentially onto the value $\omega$. In Fig. 4 we have illustrated the instantaneous frequency $d \chi / d t$ when $\omega$ is near to (but outside of) the locking region $\left|\omega-\omega_{0}\right|<\epsilon / 2 \omega_{0}$. Similarly, in Fig. 5 we have illustrated $d \chi / d t$ when $\omega$ is inside the locking region. Finally, in Fig. 6 we have illustrated the average first-order voltage response $\overline{\epsilon v^{(1)}}$ as a function of $\omega$. In Figs. 2-6 we have used the illustrative (but atypical) parameter values $A=\sqrt{2}$, $\omega_{0}=1, \epsilon=0.2, \beta_{0}=0, \alpha=\frac{1}{4} \pi$, and $\delta=0$. In addition we have used $\omega=1.5$ for Figs. 2 and 3, $\omega=1.11(\gamma=1.1)$ for Fig. 4, and $\omega=1.08(\gamma=0.8)$ for Fig. 5 .

The qualitative behavior of the other resonance solution (when $\omega \approx-\omega_{0}$ ) is very similar to that of the $\omega \approx+\omega_{0}$ solution described above. In particular, frequency locking occurs for $\tilde{\gamma}^{2}<1$ and frequency pulling occurs when $\tilde{\gamma}^{2}>1$.

Finally, let us note that the resonance solutions we have obtained are in excellent qualitative agreement with the experimental observations of Kanter and Vernon. ${ }^{3}$ Our resonance solutions clearly exhibit the phenomena of frequency locking and frequency pulling, which matches the experimental observations. Moreover, we suspect that our results

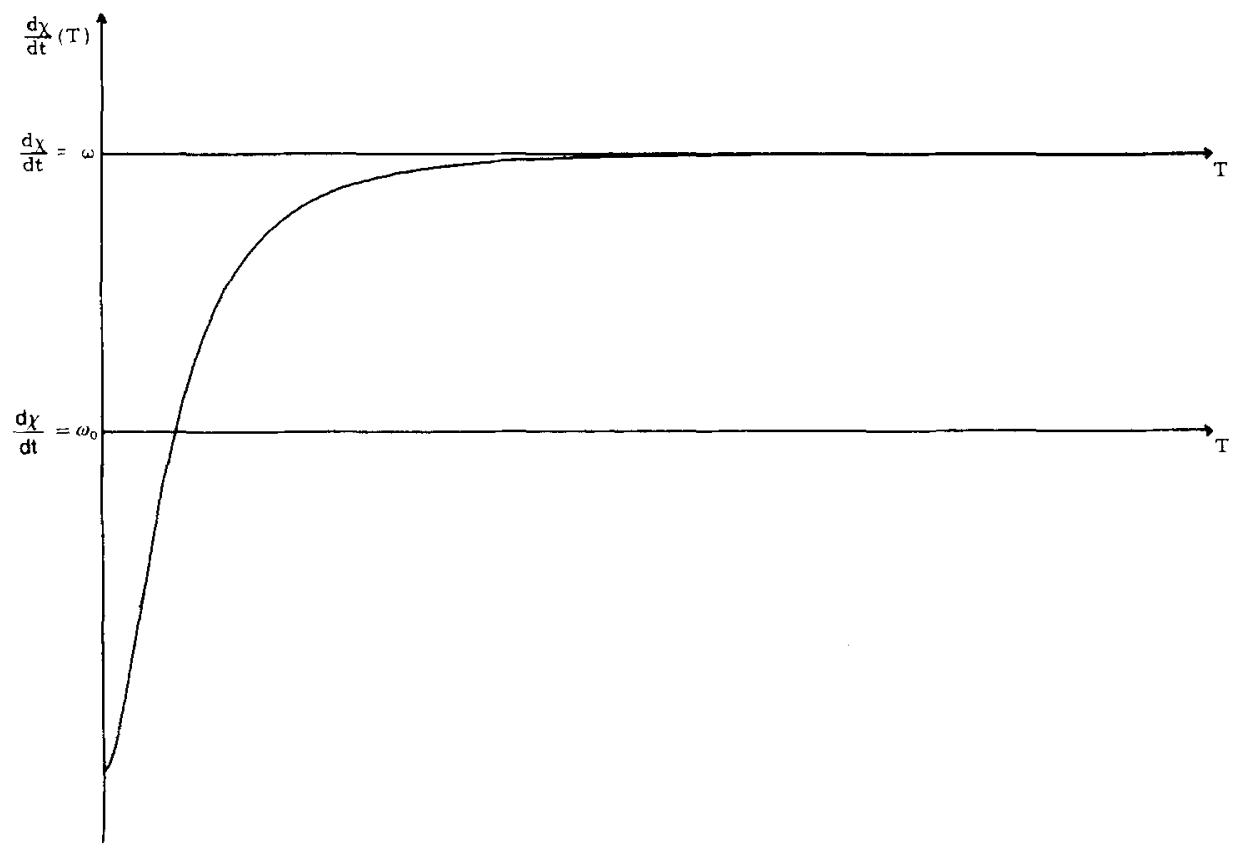

FIG. 5. The instantaneous frequency $d \chi / d t$ of the junction as a function of the slow time variable $T=\epsilon t$. Here the ac signal frequency $\omega$ is within the locking region $\left|\omega-\omega_{i}\right|<\epsilon / 2 \omega_{0}$. 


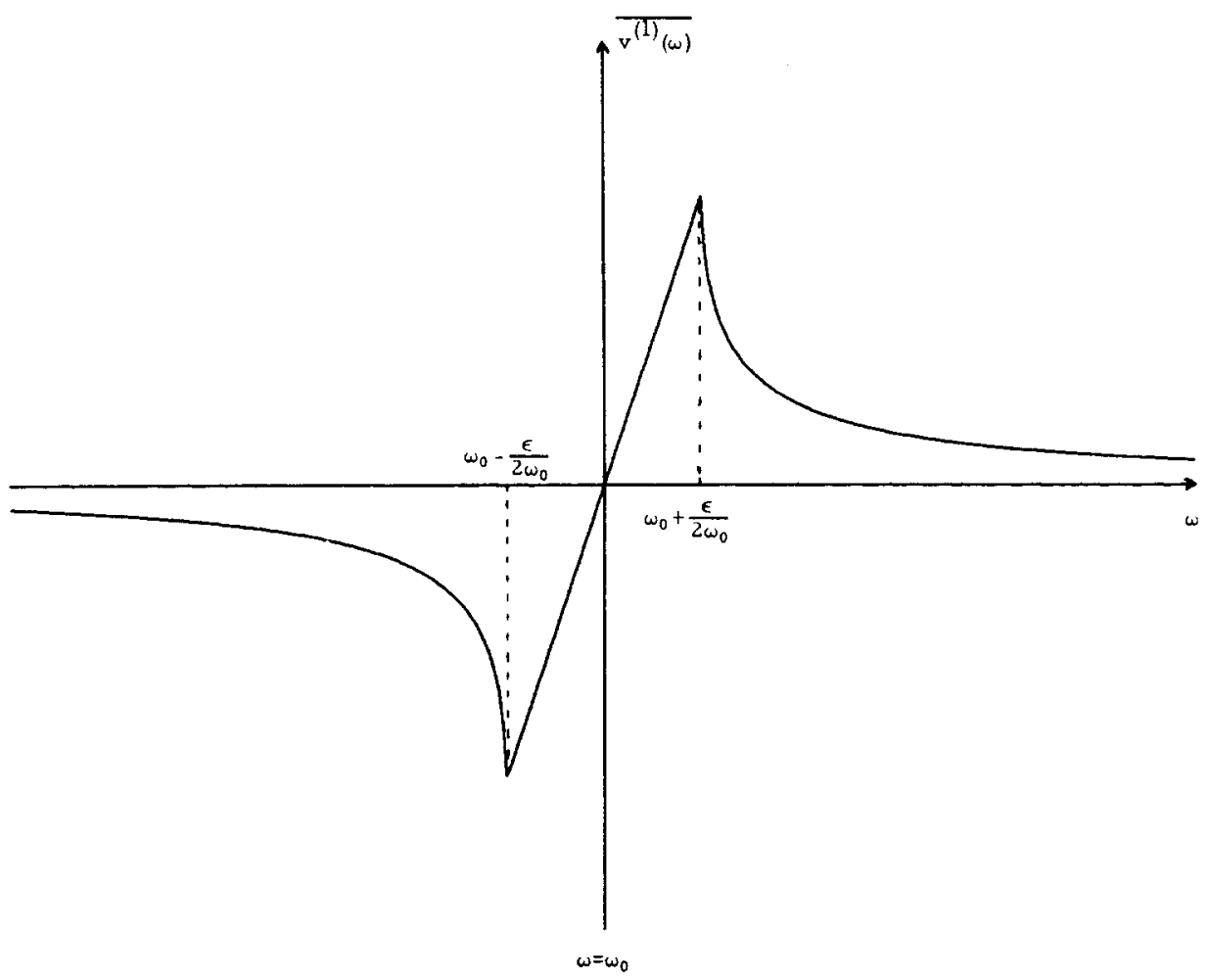

FIG. 6. The time averaged first-order response voltage $\overline{\epsilon v^{\prime \prime}}$ as a function of the ac signal frequency $\omega$.

are also in good quantitative agreement with the experimental observations, although we lack the experimental data to verify this. However note that our formulas predict that the maximum of the average first-order response voltage $\overline{v^{(1)}}$ is $\epsilon / 2 \omega_{0}$ and occurs when $\omega=\omega \pm \epsilon / 2 \omega_{0}$. This appears to be in agreement with the experimental results in Ref. 3 .

\section{SECOND-ORDER RESONANCES}

In Sec. IV we calculated expressions for $\theta$ to first order in $\epsilon$ in both the cases where first-order resonances do and do not occur. Since higher-order resonances are also of physical interest, we will now carry our calculations for $\theta$ to order $\epsilon^{2}$ in the case that no first-order resonance occurs $\left(\omega \neq 0, \pm \omega_{0}\right)$. In particular we will find that there is a general frequency shift at this order and that resonance occurs for $\omega$ near $\pm \frac{1}{2} \omega_{0}$. Similar to the first-order resonances, this secondorder resonance will cause the dominant voltage oscillation to lock its frequency onto $\pm 2 \omega$ when $\omega$ is near enough to $\pm \frac{1}{2} \omega_{0}$.

We proceed by supposing that $\omega$ is not near $0, \omega_{0}$, or $-\omega_{0}$. Then no first-order resonance occurs, and the expression in Eq. (19c) for $\theta$ remains valid on time scales of order $\epsilon t$. We, therefore, define a new slow time variable $\tau=\epsilon^{2} t$ and use the expansion

$$
\theta(\epsilon, t)=\theta^{(0)}(t, \tau)+\epsilon \theta^{(1)}(t, \tau)+\epsilon^{2} \theta^{(2)}(t, \tau)+\cdots
$$

again requiring that $\theta^{(2)}, \theta^{(3)}, \ldots$ remain of size smaller than $O\left(1 / \epsilon^{2}\right)$ for all $t$. Substitution of this expansion and the relation $d / d t=\partial / \partial t+\epsilon^{2} \partial / \partial \tau$ into the exact equation for $\theta, \mathrm{Eq}$. (14), yields the following hierarchy of equations:

$$
\frac{\partial \theta^{(0)}}{\partial t}=1
$$

$$
\begin{aligned}
\frac{\partial \theta^{(1)}}{\partial t} & \\
= & \frac{A}{\omega_{0}^{2}} \sin (\omega t-\delta)+\frac{1}{2 \omega_{0}^{2}} \sin \left(\omega t+\omega_{0} \theta^{(0)}-\alpha-\delta\right) \\
+ & \frac{1}{2 \omega_{0}^{2}} \sin \left(\omega t-\omega_{0} \theta^{(0)}+\alpha-\delta\right) \\
\frac{\partial \theta^{(2)}}{\partial t}= & \frac{1}{2 \omega_{0}}\left[\cos \left(\omega t+\omega_{0} \theta^{(0)}-\alpha-\delta\right)\right. \\
& \left.-\cos \left(\omega t-\omega_{0} \theta^{(0)}+\alpha-\delta\right)\right] \theta^{(1)}-\frac{\partial \theta^{(0)}}{\partial \tau}
\end{aligned}
$$

Solving Eqs. (47a) and (47b) as before, we find

$$
\begin{aligned}
\theta^{(0)}=t & +\beta(\tau), \\
\theta^{(1)}= & K^{(1)}-\frac{A}{\omega \omega_{0}^{2}} \cos (\omega t-\delta) \\
& -\frac{\cos \left[\left(\omega+\omega_{0}\right) t+\omega_{0} \beta(\tau)-\alpha-\delta\right]}{2\left(\omega+\omega_{0}\right) \omega_{0}^{2}} \\
& -\frac{\cos \left[\left(\omega-\omega_{0}\right) t-\omega_{0} \beta(\tau)+\alpha-\delta\right]}{2\left(\omega-\omega_{0}\right) \omega_{0}^{2}},
\end{aligned}
$$

where $K^{(1)}$ is an arbitrary integration constant to be set using the initial conditions on $\theta$, and $\beta(\tau)$ is an undetermined func- 
tion of $\tau$ to be determined at the next step in our perturbation scheme. Substituting these results into Eq. $(47 \mathrm{c})$ yields

$$
\begin{aligned}
\frac{\partial \theta^{(2)}}{\partial t}= & \left\{-\frac{d \beta}{d \tau}+\frac{1}{4\left(\omega^{2}-\omega_{0}^{2}\right) \omega_{0}^{2}}\right\}-\frac{A}{3 \omega_{0}^{3}}\{\cos [(2 \omega \\
& \left.\left.+\omega_{0}\right) t+\omega_{\delta} \beta(\tau)-\alpha-2 \delta\right]-\cos \left[\left(2 \omega-\omega_{0}\right) t\right. \\
& \left.\left.-\omega_{0} \beta(\tau)+\alpha-2 \delta\right]\right\}-\frac{1}{4\left(\omega^{2}-\omega_{0}^{2}\right) \omega_{0}^{2}} \\
& \times\left\{\cos [2(\omega t-\delta)]+\cos 2\left(\omega_{0} t+\omega_{\beta} \beta(\tau)-\alpha\right)\right\} \\
& +\frac{K^{(1)}}{2 \omega_{0}}\left\{\cos \left[\left(\omega+\omega_{0}\right) t+\omega_{0} \beta(\tau)-\alpha-\delta\right]\right. \\
& \left.-\cos \left[\left(\omega-\omega_{0}\right) t-\omega_{0} \beta(\tau)+\alpha-\delta\right]\right\} \\
& +\frac{1}{8 \omega_{0}^{3}\left(\omega-\omega_{0}\right)} \\
& \times \cos \left\{2\left[\left(\omega-\omega_{0}\right) t-\omega_{0} \beta(\tau)+\alpha-\delta\right]\right\} \\
& -\frac{1}{8 \omega_{0}^{3}\left(\omega+\omega_{0}\right)} \\
& \times \cos \left\{2\left[\left(\omega+\omega_{0}\right) t+\omega_{0} \beta(\tau)-\alpha-\delta\right]\right\}
\end{aligned}
$$

Equation (49) can immediately be integrated with respect to $t$ giving an expression for $\theta^{(2)}$. Since $\omega$ has been assumed to not be near $\omega_{0},-\omega_{0}$, or 0 , only the integrals of the quantities in the first two sets of braces in Eq. (49) can contribute unreasonably large terms to $\theta^{(2)}$. Therefore, if $\omega$ is not near $\frac{1}{2} \omega_{0}$ nor near $-\frac{1}{2} \omega_{0}$, we conclude that

$$
\frac{d \beta}{d \tau}=\frac{1}{4 \omega_{0}^{2}\left(\omega^{2}-\omega_{0}^{2}\right)}
$$

must hold for $\theta^{(2)}$ to remain bounded. In this case (no resonance at first or second orders) we thus have

$$
\beta=\frac{\tau-\tau_{0}}{4 \omega_{0}^{2}\left(\omega^{2}-\omega_{0}^{2}\right)},
$$

and this expression along with $\phi=\Phi\left(\theta^{(0)}+\epsilon \theta^{(1)}+\epsilon^{2} \theta^{(2)}+\cdots\right)$, Eqs. (48a) and (48b) for $\theta^{(0)}$ and $\theta^{(1)}$, and the integral of Eq. (49) for $\theta^{(2)}$ yield an expression for $\phi$ accurate to order $\epsilon^{2}$ and for times $t$ up to order $\left(1 / \epsilon^{2}\right)$. This expression for $\phi$ in the nonresonance case $\left(\omega \neq 0, \pm \omega_{0}, \pm \frac{1}{2} \omega_{0}\right)$ agrees with that of Kanter and Vernon. ${ }^{3}$ Note that $\beta=\left(\tau-\tau_{0}\right) / 4 \omega_{0}^{2}\left(\omega^{2}-\omega_{0}^{2}\right)$ implies that the dominant voltage term oscillates at a frequency $\omega^{*}$ given by

$$
\omega^{*}=\omega_{0}+\frac{\epsilon^{2}}{4 \omega_{0}\left(\omega^{2}-\omega_{0}^{2}\right)}+\cdots,
$$

which is shifted slightly towards the ac signal frequency.

Suppose now $\omega$ is very near to $\frac{1}{2} \omega_{0}$. We define

$$
\eta_{2} \equiv \frac{2 \omega-\omega_{0}}{\epsilon^{2}},
$$

and thus find Eq. (49) to be $\frac{\partial \theta^{(2)}}{\partial t}=\left(-\frac{d \beta}{d \tau}-\frac{1}{3 \omega_{0}^{4}}+\frac{A}{2 \omega_{0}^{4}} \cos \left\lfloor\eta_{2} \tau-\omega_{0} \beta(\tau)\right.\right.$

$$
+\alpha-2 \delta)])+ \text { nonsecular terms }
$$

where the nonsecular terms are all oscillatory in $t$ and thus cannot become unreasonably large upon integration. Since we require $\theta^{\prime 2}$ to be bounded for all $t$, from Eq. (54) we see that

$\frac{d \beta}{d \tau}=-\frac{1}{3 \omega_{0}^{4}}+\frac{A}{2 \omega_{0}^{4}} \cos \left[\eta_{2} \tau-\omega_{0} \beta(\tau)+\alpha-2 \delta\right]$

must hold. This is the key equation describing how terms of order $\epsilon^{2}$ cause order unity changes in $\phi$ over time scales of order $1 / \epsilon^{2}$, and is precisely analogous to Eq. (21) in the firstorder calculation. We now solve this equation.

Let $P(\tau)$ be defined by

$$
P(\tau)=\eta_{2} \tau-\omega_{0} \beta(\tau)+\alpha-2 \delta .
$$

Then, in terms of $P$, Eq. (55) is

$$
\frac{d P}{d \tau}=\eta_{2}+\frac{1}{3 \omega_{0}^{3}}-\frac{A}{2 \omega_{0}^{3}} \cos P
$$

The solutions to this equation are

$$
\begin{aligned}
& \tan \left(\frac{1}{2} P\right)=\frac{\left(\gamma_{2}^{2}-1\right)^{1 / 2}}{\gamma_{2}+1} \tan \left(\frac{A}{4 \omega_{0}^{3}}\left(\gamma_{2}^{2}-1\right)^{1 / 2}\left(\tau-\tau_{0}\right)\right) \\
& \tan \left(\frac{1}{2} P\right)=-\frac{\left(1-\gamma_{2}^{2}\right)^{1 / 2}}{\gamma_{2}+1} \tanh \left(\frac{A}{4 \omega_{0}^{3}}\left(1-\gamma_{2}^{2}\right)^{1 / 2}\left(\tau-\tau_{0}\right)\right) \\
& \tan \left(\frac{1}{2} P\right)=-\frac{\left(1-\gamma_{2}^{2}\right)^{1 / 2}}{\gamma_{2}+1} \operatorname{ctnh}\left(\frac{A}{4 \omega_{0}^{3}}\left(1-\gamma_{2}^{2}\right)^{1 / 2}\left(\tau-\tau_{0}\right)\right) \\
& \gamma_{2}^{2} \leqslant 1
\end{aligned}
$$

in which the definition

$$
\gamma_{2} \equiv \frac{2 \omega_{0}^{3} \eta_{2}+\frac{2}{3}}{A}
$$

has been used. As in the first-order calculation, the initial condition on $P$ (on $\beta$ ) determines not only $\tau_{0}$ but also whether Eq. $(58 \mathrm{~b})$ or $(58 \mathrm{c})$ is used in the $\gamma_{2}^{2} \leqslant 1$ case.

If $\omega$ is very near $-\frac{1}{2} \omega_{0}$, we proceed in a manner similar to the $\omega \approx \frac{1}{2} \omega_{0}$ case. This yields

$$
\begin{aligned}
& \tilde{\eta}_{2}=-\frac{2 \omega+\omega_{0}}{\epsilon^{2}}, \\
& \widetilde{P}(-\tau) \equiv \omega_{0} \beta(\tau)-\tilde{\eta}_{2} \tau-\alpha-2 \delta, \\
& \tilde{\gamma}_{2} \equiv \frac{2 \omega_{0}^{3} \tilde{\eta}_{2}+\frac{2}{3}}{A},
\end{aligned}
$$

where $P(\tau)$ is given by Eqs. (58) with $P$ and $\gamma_{2}$ replaced by $\widetilde{P}$ and $\tilde{\gamma}_{2}$.

Thus, to summarize this second-order calculation, we found that whenever $\omega$ is not near $\omega_{0},-\omega_{0}$ or 0 , we have 


$$
\phi(\epsilon, \omega, t)=\Phi(\theta(\epsilon, \omega, t)),
$$

where $\Phi(\theta)$ is defined by

$$
\tan \left[\frac{1}{2} \Phi(\theta)\right]=\frac{\omega_{0}}{A} \tan \left(\frac{\omega_{0}}{2} \theta\right)+\frac{1}{A},
$$

where

$\theta(\epsilon, t)=t+\beta(\tau)+\epsilon \theta^{\prime \prime \prime}(t, \tau)+\epsilon^{2} \theta^{(2)}(t, \tau)+O\left(\epsilon^{3}\right), \quad(61 \mathrm{c})$ and where $\theta^{\prime \prime \prime}$ is given by Eq. (48b). When $\omega \neq \omega_{0},-\frac{1}{2} \omega_{0}$ then $\beta(\tau)$ is given by

$$
\beta(\tau)=\frac{\tau-\tau_{0}}{4 \omega_{0}^{2}\left(\omega^{2}-\omega_{0}^{2}\right)}, \quad \omega \neq 0, \pm \omega_{0}, \pm \frac{1}{2} \omega_{0}
$$

and when $\omega$ is near $\frac{1}{2} \omega_{0}$ or $-\frac{1}{2} \omega_{0}, \beta(\tau)$ is given by Eqs. (53), (56), (58), and (59) or Eqs. (60) and (58), respectively.

We will now very briefly discuss these results in terms of the phase $\chi$ of the dominant term in the voltage. As before, the dominant voltage term (in reduced units) is

$$
v^{(\alpha)}=\frac{\omega_{0}^{2}}{A+\cos \left[\omega_{0} t+\omega_{0} \beta(\tau)-\alpha\right]},
$$

and its phase is

$$
\chi=\omega_{0} t+\omega_{0} \beta(\tau)-\alpha .
$$

In the nonresonance case, we use the expression for $\beta(\tau)$ in Eq. (61d) and find the phase $\chi$ is

$$
\chi=\left(\omega_{0}+\frac{\epsilon^{2}}{4 \omega_{0}\left(\omega^{2}-\omega_{0}^{2}\right)}\right) t+\text { const, } \omega \pm \pm \frac{1}{2} \omega_{0},
$$

and thus we see that the frequency of the dominant oscillation has been shifted slightly towards the ac signal frequency. In the resonance case of $\omega \approx \frac{1}{2} \omega_{0}$, we substitute expression (56) for $\beta$ into Eq. (63) and find the phase $\chi$ is

$$
\begin{aligned}
\chi & =\left(\omega_{0}+\eta_{2} \epsilon^{2}\right) t-P(\tau)-2 \delta \\
& =2 \omega t-P(\tau)-2 \delta,
\end{aligned}
$$

where $P(\tau)$ is given by Eqs. (58). Since tanh and ctnh have the limiting value 1 , we see that Eqs. (58) for $P$ imply that the small ac signal will frequency lock the voltage oscillation onto its second harmonic when $\gamma_{2}^{2} \leqslant 1$. Moreover, in this case $\left(\gamma_{2}^{2} \leqslant 1\right)$ the instantaneous frequency is

$$
\begin{aligned}
\frac{d \chi}{d t}= & 2 \omega t+\epsilon^{2} \frac{A}{2 \omega_{0}^{3}} \\
& \times \frac{1-\gamma_{2}^{2}}{\gamma_{2} \pm \cosh \left(A / 2 \omega_{0}^{3}\right)\left(\gamma_{2}^{2}-1\right)^{1 / 2}\left(\tau-\tau_{0}\right)},
\end{aligned}
$$

in which the plus or minus sign is taken according to whether $P$ is given by Eq. (58b) or (58c), respectively. Clearly the voltage oscillation frequency locks onto $2 \omega$ exponentially in $\tau=\epsilon^{2} t$

$$
\text { If } \gamma_{2}^{2} \geqslant 1 \text {, then the instantaneous frequency is }
$$

$$
\begin{aligned}
\frac{d \chi}{d t}= & \left(\omega_{0}-\frac{\epsilon^{2}}{3 \omega_{0}^{3}}\right)+\frac{\epsilon^{2} A}{2 \omega_{0}^{3}} \\
& \times\left(\gamma_{2}-\frac{\gamma_{2}^{2}-1}{\gamma_{2}+\cos \left(A / 2 \omega_{0}^{3}\right)\left(\gamma_{2}^{2}-1\right)^{1 / 2}\left(\tau-\tau_{0}\right)}\right) .
\end{aligned}
$$

This frequency oscillates about the mean frequency

$$
\begin{aligned}
\frac{\overline{d \chi}}{d t}= & \left(\omega_{0}-\frac{\epsilon^{2}}{3 \omega_{0}^{3}}\right) \\
& +\frac{\epsilon^{2} A}{2 \omega_{0}^{3}} \operatorname{sgn}\left(\gamma_{2}\right)\left[\left|\gamma_{2}\right|-\left(\gamma_{2}^{2}-1\right)^{1 / 2}\right],
\end{aligned}
$$

which makes the interpretation of frequency pulling by the second harmonic natural.

Similar phenomena occur for $\omega$ near $-\frac{1}{2} \omega_{0}$. In particular, if $\tilde{\gamma}_{2}^{2} \leqslant 1$ then the frequency of the voltage oscillation will lock onto $-2 \omega$, and if $\tilde{\gamma}_{2}^{2} \geqslant 1$, then the voltage oscillation frequency will be oscillatory in $\epsilon^{2} t$ but show a net shift towards $-2 \omega$.

We note that second-order resonant interactions have been observed. ${ }^{3}$ However, the resonance phenomena exhibited in second-order resonances have apparently not been studied experimentally.

\section{HIGHER-ORDER RESONANCES}

In principle the two-timing procedure can be extended to as high an order in $\epsilon$ as one wishes, although the algebra soon becomes overwhelming. However, the phenomena found at higher orders in the perturbation procedure will be essentially the same as the phenomena found at first and second orders. Specifically, the frequency of the junction's primary voltage oscillation, $\omega^{*}$, has (in general) terms at every even power of $\epsilon$,

$$
\omega^{*}=\omega_{0}+\epsilon^{2} \frac{1}{4 \omega_{0}\left(\omega^{2}-\omega_{0}^{2}\right)}+\epsilon^{4} \omega_{4}+\cdots
$$

when no resonance occurs. Moreover, resonances can occur wherever a multiple of $\omega^{*}$ is near a harmonic of the ac signal. Wherever a resonance does occur, it will cause frequency locking and frequency pulling such as occurred at first and second orders.

For example, one would expect that third-order resonances would occur whenever $\omega$ is near $\pm \frac{1}{3} \omega^{*}, \pm \frac{2}{3} \omega^{*}$, $\pm 2 \omega^{*}$, or $\pm 3 \omega^{*}$. However, detailed calculations show that resonance occurs only when $\omega$ is near $+\frac{1}{3} \omega^{*}$ or near $-\frac{1}{3} \omega^{*}$. Moreover, when $\left|3 \omega-\omega^{*}\right|<(9 / 128)\left[\left(1+8 A^{2}\right) / \omega_{0}^{5}\right] \epsilon^{3}$, then the ac signal will frequency lock the primary voltage oscillation onto the frequency $3 \omega$. Similarly the primary oscillation will frequency lock onto $-3 \omega$ if $\left|3 \omega+\omega^{*}\right|<(9 / 128)$ $\times\left[\left(1+8 A^{2}\right) / \omega_{0}^{5}\right] \epsilon^{j}$. When $\omega$ is near to (but outside of) these frequency locking regions, the voltage oscillation will be frequency "pulled" toward the appropriate of $3 \omega$ or $-3 \omega$.

Although one can extend these calculations to higher order, extremely high-order calculations are often not of much physical interest. One notes that in calculating the effects of an $n$ th-order resonance, one must require that the ac signal's frequency bandwidth be of a smaller size then $O\left(\epsilon^{n}\right)$. This is a physically sensible requirement since it is 
equivalent to requiring that the ac signal lifetime be of a size longer than $O\left(1 / \epsilon^{n}\right)$, which is the time scale on which the $n$ th-order resonance phenomena (e.g., frequency locking) manifest themselves.
'D.B. Sullivan, R.L. Peterson, V. Kose, and J.E. Zimmerman, J. Appl. Phys. 41, 4865 (1970).

${ }^{2}$ N.R. Werthamer and S. Shapiro, Phys. Rev. 164, 523 (1967).

'H. Kanter and F.L. Vernon, Jr., J. Appl. Phys. 43, 3174 (1972)

'J.C. Neu, thesis (California Institute of Technology, 1978) (unpublished). 